\title{
Neuronal Response Latencies Encode First Odor Identity Information across Subjects
}

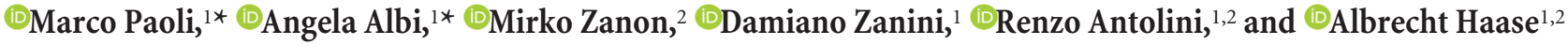 \\ ${ }^{1}$ Center for Mind/Brain Sciences, University of Trento, Rovereto 38068, Italy and ${ }^{2}$ Department of Physics, University of Trento, Trento 38120, Italy
}

Odorants are coded in the primary olfactory processing centers by spatially and temporally distributed patterns of glomerular activity. Whereas the spatial distribution of odorant-induced responses is known to be conserved across individuals, the universality of its temporal structure is still debated. Via fast two-photon calcium imaging, we analyzed the early phase of neuronal responses in the form of the activity onset latencies in the antennal lobe projection neurons of honeybee foragers. We show that each odorant evokes a stimulus-specific response latency pattern across the glomerular coding space. Moreover, we investigate these early response features for the first time across animals, revealing that the order of glomerular firing onsets is conserved across individuals and allows them to reliably predict odorant identity, but not concentration. These results suggest that the neuronal response latencies provide the first available code for fast odor identification.

Key words: calcium imaging; honeybee; latency coding; odor coding; olfation; two-photon microscopy

\section{Significance Statement}

Here, we studied early temporal coding in the primary olfactory processing centers of the honeybee brain by fast imaging of glomerular responses to different odorants across glomeruli and across individuals. Regarding the elusive role of rapid response dynamics in olfactory coding, we were able to clarify the following aspects: (1) the rank of glomerular activation is conserved across individuals, (2) its stimulus prediction accuracy is equal to that of the response amplitude code, and (3) it contains complementary information. Our findings suggest a substantial role of response latencies in odor identification, anticipating the static response amplitude code.

\section{Introduction}

Odorant perception initiates when an odorant molecule interacts with the olfactory receptors (ORs) on the dendrites of the olfactory receptor neuron (ORN; Mombaerts et al., 1996). The nature of odorant-receptor interaction allows for a certain odorant to bind to multiple receptors with different affinities (Münch and Galizia, 2016), which results in the activation of multiple ORNs with different response intensities and latencies. These signals converge into the antennal lobe $(\mathrm{AL})$, whose functional units, called glomeruli, receive input from a single ORN type, process this information through a network of local interneurons, and

\footnotetext{
Received Feb. 19, 2018; revised Aug. 10, 2018; accepted Aug. 15, 2018.

Author contributions: M.P. and A.H. designed research; M.P., A.A., M.Z., and D.Z. performed research; R.A. and A.H. contributed unpublished reagents/analytic tools; M.P., A.A., and A.H. analyzed data; M.P. and A.H. wrote the paper.

This work was supported by the University of Trento (Grant P.S.17/21-BRANDY) and the Autonomous Province of Bolzano (Project B26J16000310003). We thank Paul Szyszka and Stefano Panzeri for useful discussions.

*M.P. and A.A. contributed equally to this work.

The authors declare no competing financial interests.

Correspondence should be addressed to either of the following: Albrecht Haase at the above address, E-mail:

albrecht.haase@unitn.it; or Marco Paoli at the above address, E-mail: marco.paoli@uni-konstanz.de.

DOI:10.1523/JNEUROSCI.0453-18.2018

Copyright $\odot 2018$ the authors $\quad 0270-6474 / 18 / 389240-12 \$ 15.00 / 0$
}

relay the resulting information via projection neurons (PNs) to higher brain areas (Galizia, 2014). Fundamental studies have shown that information about the odor stimulus identity and intensity is encoded in the mean response amplitudes of the glomerular PNs (Sachse and Galizia, 2003) and that this static spatial code is conserved across individuals (Galizia et al., 1999b). At the same time, stimulus-related information is not restricted to the spatial code, but temporal features may also contribute to odor coding (Laurent et al., 1996; Spors and Grinvald, 2002).

A robust measure of neural response delay is first spike latency (Chase and Young, 2007; Junek et al., 2010). It can successfully predict stimulus identity in different sensory modalities, including auditory (Furukawa and Middlebrooks, 2002; Nelken et al., 2005), visual (Gawne et al., 1996; Masquelier, 2012; Reich et al., 2014), and somatosensory (Panzeri et al., 2001; Petersen et al., 2001, 2002) systems. An involvement of neuronal response latency in odor coding was first proposed and modeled (Hopfield, 1995; Margrie and Schaefer, 2003) and later experimentally tested in vertebrates (Spors and Grinvald, 2002; Abraham et al., 2004; Wilson et al., 2017) and invertebrates (Müller et al., 2002; Krofczik et al., 2008). A pioneering electrophysiological study in the locust's antennal lobe revealed that the spatiotemporal patterns of odor-induced 
activity across a PN population allows predicting stimulus identity and intensity (Stopfer et al., 2003). The following studies confirmed that OR response latencies are odorant specific and conserved across repeated stimulations: Spors et al. (2006), Junek et al. (2010), and Martelli et al. (2013). Moreover, the stereotyped $\mathrm{AL}$ (or olfactory bulb) topology supports the possibility that response latencies might be preserved across subjects (Ressler et al., 1994; Galizia et al., 1999b). Patterns of glomerular responses are dynamic, and sensory information can be encoded by the spatial representation of the active neurons and also by temporal features such as response latency, rise time, temporal profile, and oscillatory activity (Stopfer et al., 2003; Schaefer and Margrie, 2007; Brill et al., 2013; Grillet et al., 2016; Paoli et al., 2016b). Nevertheless, the contribution of spatial and temporal features to olfactory coding is still debated. Although latency patterns of odorant-elicited responses in single Xenopus laevis larvae were shown to reliably identify odor stimuli (Junek et al., 2010), another study showed that firing latencies are less informative than response intensity maps (Bathellier et al., 2008).

The present study probes whether stimulus-related response latencies are conserved across individuals and whether they provide sufficient information to identify an odorant. Functional calcium imaging was used to characterize the response patterns of five odorants across the AL output neurons, suggesting that odorant classification can be correctly performed based on glomerular response latency distribution. By analyzing the order at which glomeruli are activated, we show the existence of stimulusspecific response latency maps that are conserved across animals, providing information that is available early and with a prediction accuracy comparable to the response amplitude code. Finally, we test the associative learning and generalization scores for the same odorants using the proboscis extension reflex (PER) paradigm (Takeda, 1961; Bitterman et al., 1983; Giurfa and Sandoz, 2012). We were able to observe that bees were capable of discriminating most of the learned stimuli from the unconditioned ones, although failing on those, which also do not provide a reliable code at the neural level.

\section{Materials and Methods}

Animal preparation. Foragers honeybees, Apis mellifera, were collected from outdoor beehives the day before the experiments and housed in insect tents (BugDorm-2120) under the supply of a $50 \%$ sucrose/water solution. Animal preparation for calcium imaging was performed as described previously (Sachse and Galizia, 2002; Paoli et al., 2016b). After capture, bees were fixed in a custom-made Plexiglas holder that allows fixing the head with soft dental wax. To proceed with staining, a small window in the head cuticle was opened and glands and tracheas were displaced to expose the injection site. The tip of a borosilicate glass needle coated with Fura-2-dextran (Thermo Fisher Scientific) was inserted between the medial and lateral mushroom body calyces, where medial antennocerebral tracts (m-ACTs) and lateral antennocerebral tracts (1-ACTs) cross. After dye injection, the head capsule was closed to prevent brain desiccation, and bees were fed ad libitum with a 50\% sucrose/ water solution. On the following day, antennal lobes were exposed to allow optical access, and the brain was covered in transparent twocomponent silicon (Kwik-Sil, WPI). A similar amount of left and right ALs were prepared to balance eventual lateralization effects (Haase et al., 2011a; Rigosi et al., 2011, 2015). In this procedure, the degree of labeling depends on the precision of the injection site and on the amount of dye loaded on the glass capillary. Hence, a variable level of PN labeling may result. Nonetheless, the reproducibility of response amplitudes and the lack of labeling bias for specific glomeruli indicate that the loading procedure is reproducible.

Stimulus generation. Olfactory stimulation was performed with a custom-built, computer-controlled olfactometer comprising seven inde- pendently controlled odor channels (for details, see Paoli et al., 2017). Fast microvalves switched between odorants with a temporal precision of $<2 \mathrm{~ms}$. Continuous air suction behind the bee cleared residual odor traces. The device was controlled by a LabVIEW-programmed user interface, and synchronization of stimulus delivery and image acquisition was controlled with a clock rate of $2 \mathrm{kHz}$. Five odorants were used in this study: 1-hexanol, 3-hexanol, 1-nonanol, isoamyl acetate, and acetophenone (Sigma Aldrich). All selected odorants are ecologically relevant, had been used previously to study olfactory coding in honeybees, and induce a clear response in the antennal lobe at the chosen dilution (Galizia et al., 1999b; Szyszka et al., 2011; Paoli et al., 2016a). In addition, they vary in several structural and functional parameters: 1-hexanol and 1-nonanol are alcohols with different chain length, whereas 1- and 3-hexanol differ only by the hydroxyl group position. As a contrast to the three alcohols, the structurally different aromatic ketone acetophenone was tested. Finally, isoamyl acetate, a component of the bee alarm pheromone, was used in contrast to the floral odors. Samples were prepared fresh every week at a 1:200 (or 1:2000) dilution in mineral oil. We found the dilution of 1:200 to be well above the bee's detection threshold and able to activate multiple glomeruli without saturating olfactory receptors.

After imaging, odor stimulus concentration and kinetics at the honeybee's antennae were measured with a fast photo-ionization detector (PID; miniPID 200, Aurora) to determine stimulus arrival time at the olfactory receptors on the antenna in our experimental conditions. The analysis of the PID data is based on five repeated $1 \mathrm{~s}$ stimuli generated for each odorant with the olfactometer of the imaging setup. To determine stimulus arrival time at the antennae, we first measured the odordependent propagation time within the PID and then subtracted it from the PID curves. Then, we determined the mean arrival times and their fluctuations at the point of the bee antennae for the different odor stimuli. To prevent confusion, we still present all imaging data with respect to an absolute time scale, where $t=0$ represents the moment of the computer-controlled valve opening.

The PID signal amplitude allows us to determine the absolute odorant concentration and monitor reproducibility of the odor stimulus profile. Although odorant dilutions are identical (1:200), their concentration in the vial's headspace varies because of the odor-specific vapor pressures (1-hexanol, $126 \mathrm{~Pa}$; 3-hexanol, $450 \mathrm{~Pa}$; 1-nonanol, 5.46 Pa; isoamyl acetate, $747 \mathrm{~Pa}$; acetophenone, $52.9 \mathrm{~Pa}$; all at $25^{\circ} \mathrm{C}$ ). With the calibration curves provided by the PID manufacturers, we converted odordependent PID voltages into absolute concentrations. At the end of the stimulus period, when the PID curves are about to saturate (see Fig. $3 E$ ), the following approximate concentrations apply: 1-hexanol, $1.4 \mathrm{ppm}$; 3-hexanol, $3.1 \mathrm{ppm}$; 1-nonanol, $0.054 \mathrm{ppm}$; isoamyl acetate, $1.5 \mathrm{ppm}$; acetophenone, $0.036 \mathrm{ppm}$.

Two-photon functional imaging. Optical imaging was performed, as described previously (Haase et al., 2011b), via two-photon laserscanning microscopy with an imaging platform based on an Ultima IV microscope (Bruker) optimized for in vivo insect imaging (Haase, 2011). The calcium-sensitive dye Fura-2-dextran was excited at $800 \mathrm{~nm}$. A high temporal resolution was obtained via repetitive scanning of onedimensional (1D) line traces across all glomeruli of interest at a rate of $100 \mathrm{~Hz}$. The calcium-induced fluorescence changes were recorded with a photomultiplier (Hamamatsu) in a filter window of $525 \pm 20 \mathrm{~nm}$. Calcium imaging monitors the change in an intracellular calcium concentration, which is an indirect indicator of neuronal activity. Hence, the observed signal is delayed with respect to the action potential (Homma et al., 2009). Even if calcium imaging allows detecting weak activities down to individual action potentials (Homma et al., 2009; Grewe et al., 2010), in our setup the signal-to-noise ratio is limited by the short pixel exposure time because of spatial scanning. Therefore, we could not detect single spikes, and even very weak burst responses may have been missed.

Postprocessing of imaging data. Single glomeruli were identified along the $1 \mathrm{D}$ line scans using a 2D high-resolution reference image. Glomerular response curves $F(t)$ synchronized with the odor stimuli were extracted and averaged over the three recorded trials for each odor and each bee. These fluorescence curves were normalized with respect to their mean prestimulus baseline activity $F_{\mathrm{b}}$, providing the relative fluorescence change $\Delta F / F(t)=\left(F(t)-F_{\mathrm{b}}\right) / F_{\mathrm{b}}$. 
Briefly, the onsets of glomerular activity were determined in the following way. First, the baseline activity was analyzed during the $500 \mathrm{~ms}$ window before stimulus onset, obtaining mean value and SD of the normally distributed fluorescence. Then, single glomeruli were classified as responsive when the fluorescence after the stimulus onset stayed for $>80$ ms above a threshold of 0.66 of the cumulative probability distribution of baseline activity. The response latency was then approximated by the first time point of this activity period. In the 25 animals, each tested to five odorants, a total of 700 glomerular responses were identified according to this criterion. To maximize the detection of stimulus-induced activities, the low threshold described above was applied to the activity detection algorithm. On the other hand, this approach likely identified a non-negligible amount of spontaneous activity as odor related. This adds noise to the system, influencing the predictive power of the latency code.

Correlation of response latencies and amplitudes. For each odor, the distribution of glomerular response latencies was analyzed via fitting Gaussian curves to the peak of the early response. The normal distributed early responses $(<200 \mathrm{~ms})$ and the uniformly distributed late responses ( $>200 \mathrm{~ms}$ ) were separately analyzed for potential correlations between response latency and response amplitude using Pearson's correlation coefficients.

Testing odor specificity and generality of the latency code: general concept of the analysis. The analysis of glomerular response latencies is constrained by the fact that absolute response latency variations are dominated by odor- and glomerulus-independent offsets across individuals. To avoid these biases, olfactory neuronal responses are classified by the order in which they are triggered in single subjects, rather than by the absolute onset timing. Hence, for each animal, the odor-induced response is represented by an odor-specific latency rank vector. By the use of relative latency vectors, the absolute delay of the intracellular calcium increase with respect to the changes in membrane potential has no influence on the rank code. However, this code is not equivalent to a first spike code, which is based on absolute spike timing, but rather reflects the order of firing onsets (Thorpe et al., 2001).

The similarity between any two latency vectors is tested and quantified by the rank correlation coefficient (RCC; Eqs. 1, 2). To assess whether the latency rank code is odor specific and conserved across individuals, responses of individual test animals (i.e., individual latency vectors; Eq. 3) are compared with the average latency vectors of all remaining animals (odor template vectors; Eq. 4). After selecting an odor response vector of a single test bee in a stimulus-blind mode, the eliciting odorant is predicted from the maximum correlation (Eq. 5) between this test latency vector and the odor template vectors. A prediction probability of the latency code is obtained by repeating this analysis for each of the 25 bees.

To compare odor specificity and generalizability of the latency code with the amplitude code, the same analysis is performed on the response amplitude by creating an amplitude rank vector. Furthermore, we test the redundancy of the information of both parameters by combining both codes and by comparing the prediction accuracy to latency and amplitude separately. Finally, the neural codes are compared with behavioral odor generalization in a classical conditioning experiment. The single analysis steps are described in detail in the following paragraphs.

Rank correlation coefficient. To evaluate odorant specificity and generality of response latencies, cross-correlations of glomerular response latencies were calculated between different odorant responses in different bees. Single responses were characterized by a response vector, containing the response latencies for all activated glomeruli. For pairwise comparisons between different stimuli and different bees, only commonly activated glomeruli were considered, to avoid any bias from the spatial response pattern on the temporal latency code.

The characterization of odor responses was restricted to the rank of onset of single glomeruli (Junek et al., 2010). To compare two responses, $X$ and $Y$, instead of looking at the correlation of absolute latencies, we used Kendall's RCC, $\tau$ (Kendall, 1938). This can be expressed by the number of sorting inversions $n_{\text {inv }}$ required to establish in vector $X$ the same latency order as in vector $Y$, or vice versa. Normalization by the maximum number of inversions and projection onto the interval $[-1,1]$ gives the following:

$$
\tau(X, Y)=1-\frac{2 n_{\text {inv }}}{n_{\text {act }}\left(n_{\text {act }}-1\right) / 2} .
$$

$\tau$ becomes 1 for the perfect positive correlation, 0 is the absence of the correlation, and -1 is the perfect negative correlation.

Rank correlation coefficients are now calculated across animals for different odor stimuli. Results are therefore labeled by four parameters: for odorant $A$ applied to bee $k$ and odorant $B$ applied to bee $m$, the rank correlation coefficient becomes as follows:

$$
\tau(A, k, B, m)=1-\frac{2 n_{\text {inv }}(A, k, B, m)}{n_{\text {act }}(A, k, B, m)\left(n_{\text {act }}(A, k, B, m)-1\right) / 2},
$$

where only those glomeruli showing activity in both response vectors are compared. The number of commonly activated glomeruli is given by $n_{\text {act }}(A, k, B, m)$.

Odorant predictability. The generalizability of a latency rank code was tested by analyzing stimulus predictability. Single bees were chosen as test subjects, represented by their response latency vectors for the different tested odorants. To compare these test responses against a potential universal latency code, odorant template vectors were created from the responses of all remaining animals of the cohort. To obtain these templates, single response latency vectors were corrected against odorantindependent delays by projecting latencies linearly onto the interval $[0$, 1]. Such a normalized latency vector in response to odorant $A$ in bee $k$ is obtained from the following:

$$
X_{\text {norm }}(A, k)=\frac{\left(x_{1}, x_{2}, \ldots, x_{\mathrm{n}}\right)-\min x_{\mathrm{j}}}{\max x_{\mathrm{j}}-\min x_{\mathrm{j}}},
$$

where $x_{\mathrm{j}}$ is the single glomerular response latency.

To create a template vector for odorant $A$, normalized responses were then averaged over all bees except the test subject $k$ via weighted averaging. The weights were the numbers of commonly active glomeruli $n_{\text {act }}(A$, $k, i)$, which reward higher information content when more glomeruli can be compared. The template vector for an averaged response to odorant $A$ involving all animals except the test bee $k$ is then given by the following:

$$
X_{\text {temp }}(A, k)=\frac{\sum_{\mathrm{i} \neq \mathrm{k}} n_{\text {act }}(A, k, i) X_{\text {norm }}(A, i)}{\sum_{\mathrm{i} \neq \mathrm{k}} n_{\text {act }}(A, k, i)} .
$$

To quantify the correlation between a test subject's response and these average response vectors, the five rank correlation coefficients between the test latency vector and the odorant templates were calculated. To determine the odorant predictability, this procedure was repeated for each of the 25 bees as a test subject, providing as a result 25 rank correlation coefficient matrices of dimension $5 \times 5$, containing the correlations of all test odorants with all template odors in every single bee. To predict a single test odor $A$ in a single bee $k$, we calculated the maximum rank correlation coefficient $\tau_{\max }$ between the test response $X_{\text {test }}(A, k)$ and the five template odorants $B_{i}$ :

$$
\tau_{\max }\left(X_{\text {test }}(A, k)\right)=\max _{B_{\mathrm{i}}} \tau\left(X_{\text {test }}(A, k), X_{\text {temp }}\left(B_{\mathrm{i}}, k\right)\right) .
$$

Averaging the prediction results over all bees produces a generalization matrix (Quian Quiroga and Panzeri, 2009) showing prediction probabilities of all template odors for each test odorant.

Amplitude code and combined code. To compare the latency code with the static response amplitude code, we calculated the latter from the imaging data by averaging the relative fluorescence change in single glomeruli over $800 \mathrm{~ms}$ after their response onset. Amplitude rank vectors for the single bee responses were then formed by the ordered amplitudes of responsive glomeruli. As for the latency code, the comparison of single amplitude response vectors was performed via the rank correlation coefficient (Eq. 1). To quantify odor predictability via the amplitude rank code, single test bee responses are compared with 
template vectors (Eq. 4) calculated by averaging the normalized amplitude rank vectors weighted by the number of activated glomeruli (Eq. 3) of all remaining bees. As for the latency code, the tested odorant is predicted by the highest RCC between test and template vectors for the five odorants (Eq. 5). The mean prediction probabilities for each template odor against the test odorants generate the generalization matrix of the amplitude code.

To test whether there is complementary information in latency and amplitude code, both codes were combined by comparing the RCC values of test and template vectors not separately for latencies and amplitudes, but by choosing the highest RCC across all 10 confrontations of a test latency vector with the latency templates and a test amplitude with the amplitude templates. The predicted odor by this combined code is then averaged again over all tests, which forms the generalization matrix for a combined code.

Behavioral generalization via a proboscis extension reflex assay. For the PER assay, bees were briefly ice-anesthetized and restrained in individual stages with the head blocked with a tape strip (Bitterman et al., 1983). Bees were fed with a $50 \%$ sucrose/water solution, and experiments were performed $\sim 2 \mathrm{~h}$ after the animals' preparation. A LabVIEW-based software controlled the olfactometer (analogous to the one used for imaging, implemented for semiautomatic PER assays), which determined odor presentation onset, duration, and intertrial interval. Odorants were diluted 1:200 in mineral oil to match imaging conditions. The conditioning procedure consisted of five pairings between odorant [conditioned stimulus (CS)] and sucrose reward [unconditioned stimulus (US)]. During conditioning, each animal was individually exposed for $28 \mathrm{~s}$ to a flow of clean air (context familiarization), followed by $4 \mathrm{~s}$ of an initially neutral odorant. A 50\% sucrose/water solution was presented as a reward for $3 \mathrm{~s}$ with a $1 \mathrm{~s}$ temporal overlap between the CS and US. After each conditioning trial, each bee was kept in place for an additional $28 \mathrm{~s}$ and then removed from the airflow. Proboscis extension after CS presentation and before US presentation was checked for establishing the learning curve. Only an extension beyond the virtual line between the open mandibles was counted as a positive response. After successful conditioning, animals were tested against all odorants to generate the behavioral generalization matrix (Guerrieri et al., 2005). To reduce extinction probability of learned responses, the protocol was shortened to $13 \mathrm{~s}$ prestimulus and poststimulus clean air exposure, $4 \mathrm{~s}$ of test odorant exposure, and $5 \mathrm{~min}$ of the intertest interval. Bees were trained in groups of 10, and test odorants were presented in a randomized order by the stimulus generator in an investigator-blind way. The final analysis was performed on all bees that learned the odor and responded to at least one of the five test odorants: $n=26$ animals trained for 1-hexanol; $n=26$ for 3-hexanol; $n=29$ for 1-nonanol; $n=23$ for isoamyl acetate; and $n=25$ for acetophenone.

Experimental design and statistical analysis. The significance of the results predicting a test odorant via the maximum rank correlation coefficient $\tau_{\max }$ was determined by a $\chi^{2}$ test for each odor on the null hypothesis that predictions were random, which would produce each of the five outcomes with a probability of $20 \%$. Results were Bonferroni corrected for the familywise error rate.

The differences in the average prediction accuracies among latency, amplitude, and combined code were tested for significance with paired $t$ tests on $n=125$ samples ( 5 odors $\times 25$ bees). Test results were corrected for a false discovery rate via the Benjamini-Hochberg method.

In the PER experiments, for each trained group, differences between the response to the trained odorant and each of the four novel stimuli were tested with multiple $t$ test tests, group sizes as indicated in the previous section. Results were Bonferroni corrected for the familywise error rate.

The overlap between neural codes and the behavioral code was quantified with a $\chi^{2}$ goodness-of-fit test. The observed prediction probabilities of the neural codes were confronted with the behavioral generalization probabilities for all odor test/template combinations, for which the PER responses were nonzero (19 odor combinations).

To analyze the influence of concentration on the latency rank code, a bootstrapping analysis (10,000 samples with replacements) was performed on the mean rank correlation coefficient $\bar{\tau}$ averaged over 25 test bees:

$$
\begin{aligned}
& \bar{\tau}\left(X_{\text {test }}(A), X_{\text {temp }}(B)\right) \\
& =1-\frac{\sum_{\mathrm{k}=1}^{\mathrm{N}_{\text {bee }}} 2 n_{\text {inv }}\left(X_{\text {test }}(A, k), X_{\text {temp }}(B, k)\right)}{\sum_{\mathrm{k}=1}^{\mathrm{N}_{\text {bee }}} \max _{\mathrm{j} \neq \mathrm{k}}\left[n_{\text {act }}(A, B, j)\left(n_{\text {act }}(A, B, j)-1\right)\right] / 2} .
\end{aligned}
$$

The set of test bees were sampled with replacements $(10,000$ samples for each stimulus). The resulting bootstrap distributions of $\bar{\tau}$ were compared for different concentrations and different odorants.

\section{Results}

\section{Spatial distribution of olfactory response activity}

Honeybee antennal lobe PNs were labeled with a calciumsensitive dye (Paoli et al., 2017) to optically measure odorantinduced intracellular calcium variations. Spatiotemporal response patterns were recorded for five odorants in 25 bees. Figure $1 A$ shows a single glomerulus resolved by the two-photon excited fluorescence signal. We recorded neural activities from 12 glomeruli of similar imaging planes. Glomeruli were identified by comparing their morphology and anatomical location with the honeybee AL reference atlas (Galizia et al., 1999a; Fig. 1B). For each odorant, glomerular responses were obtained from stimulusevoked relative fluorescence changes, averaged over time and over three successive odorant presentations (Fig. 1C). The mean activity odor response maps show odorant-specific activations, which are highly conserved across individuals. Each glomerulus was activated by one to three of the five odorants, and each odorant induced a strong excitatory response in two to four of the monitored glomeruli (Fig. 1C).

\section{Odorant-dependent response latencies}

Glomerular responses vary in intensity, duration, onset, and rise time. Nonetheless, their temporal response profiles are highly conserved not only within trials but also across individuals, and they produce very homogeneous patterns even after averaging across all animals (Fig. 2A). Two examples of highly conserved response latencies are presented in Figure 2, $B$ and $C$. In particular, a selective and consecutive activation of glomeruli 17 and 33 after nonanol stimulation is shown for three honeybees, where glomerulus 17 displays a consistently shorter response latency compared with glomerulus 33 (Fig. 2B). Similarly, glomeruli 48 and 36 are both activated by isoamyl acetate with similar response amplitude, but glomerulus 36 consistently anticipates the response onset of glomerulus 48 (Fig. 2C). Overall, odorant-evoked glomerular responses appear to be conserved across animals, and both stimulus and glomerulus-specific. Moreover, Figure 2-1 (available at https://doi.org/10.1523/JNEUROSCI.0453-18.2018. f2-1) shows that the responses of the 25 bees are highly conserved, which confirms that, although dye loading variability may exist, the overall response is consistent across animals both in terms of response amplitude and kinetics.

To determine odorant-related responses, we used a thresholding algorithm. A response was defined as a long-lasting deviation from the mean resting state that was temporally correlated with the stimulus. With this method, we were able to identify 700 stimulus-correlated glomerular responses across all analyzed bees. Notably, calcium imaging represents an indirect measure of a change in voltage potential associated with a spiking activity. Nonetheless, because of the fast increase in intracellular calcium concentration after neuronal activity and to the fast Fura-2 calcium binding kinetics, this method allows, although with a constant offset to electrophysiology (Junek et al., 2010), a precise and reliable detection of both induced and spontaneous activity. On the contrary, slow Fura-2 calcium dissociation kinetics and slow 
A

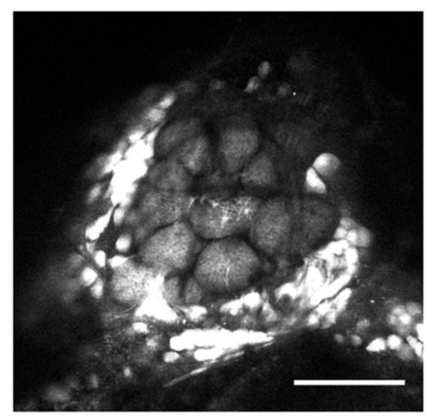

B

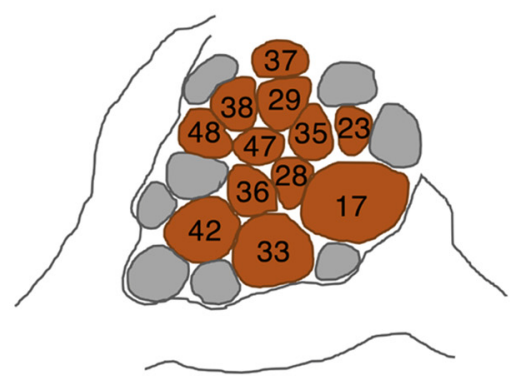

C

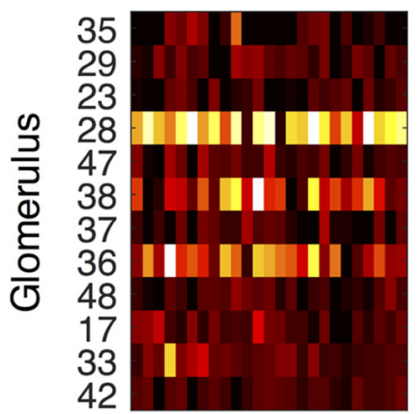

Bees

isoamyl acetate

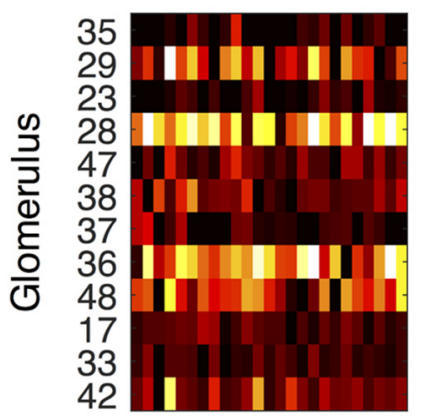

Bees 3-hexanol

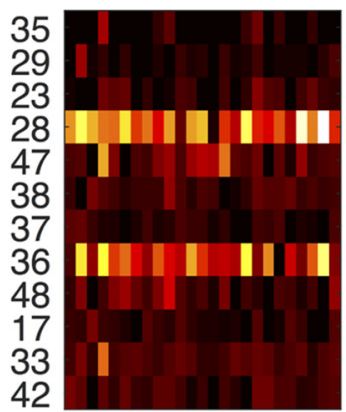

Bees

\section{acetophenone}

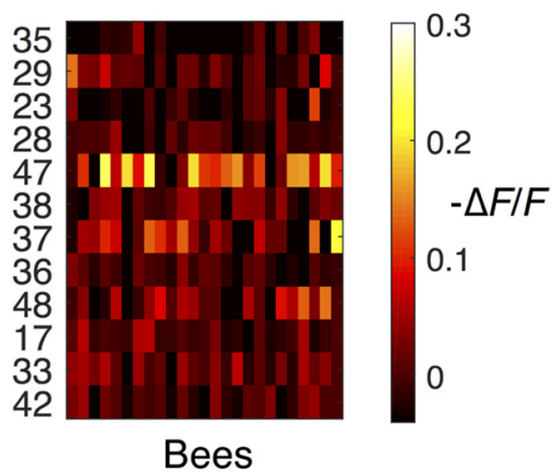

Figure 1. Spatial odorant response maps. $\boldsymbol{A}, 0$ ptical section of an antennal lobe. Projection neurons are backfilled with Fura-2-dextran. Scale bar, $100 \mu \boldsymbol{m}$. $\boldsymbol{B}$, Glomeruli in $\boldsymbol{A}$ have been identified and labeled: brown, glomeruli identified across all animals and used for the analysis; gray, glomeruli excluded from the analysis. $\boldsymbol{C}$, Time- and trial-averaged responses as a function of odorant, glomerulus ( $y$-axis), and subject ( $x$-axis). The mean values are calculated over the $200-600$ ms window after stimulus onset and over three repeated trials. Each odorant induces the activation of a glomerular pattern that is highly conserved across bees. $-\Delta F / F$ indicates the change in fluorescence normalized to background fluorescence.

A

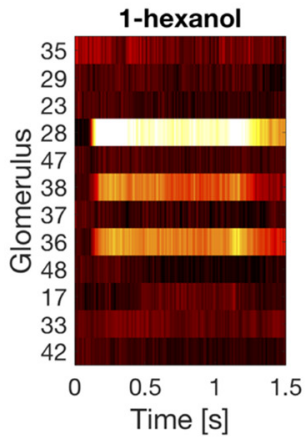

B

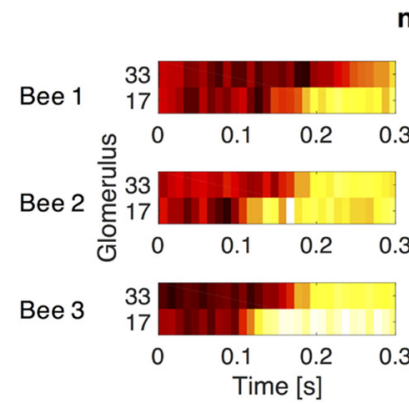

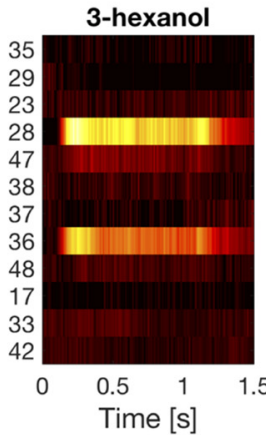

nonanol
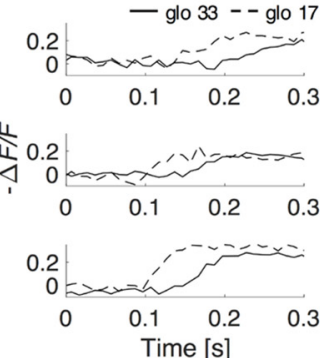

nonanol

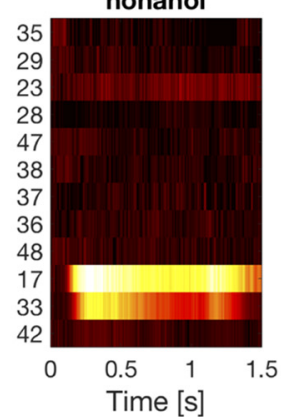

C

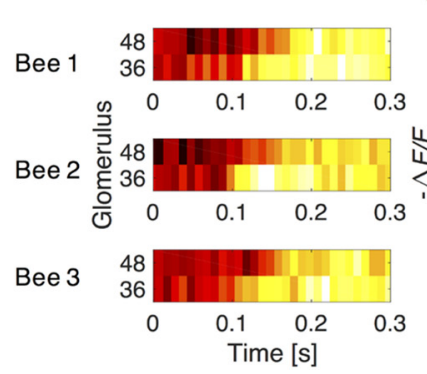

nonanol

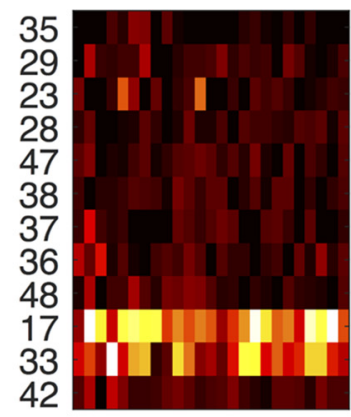

Bees 
A
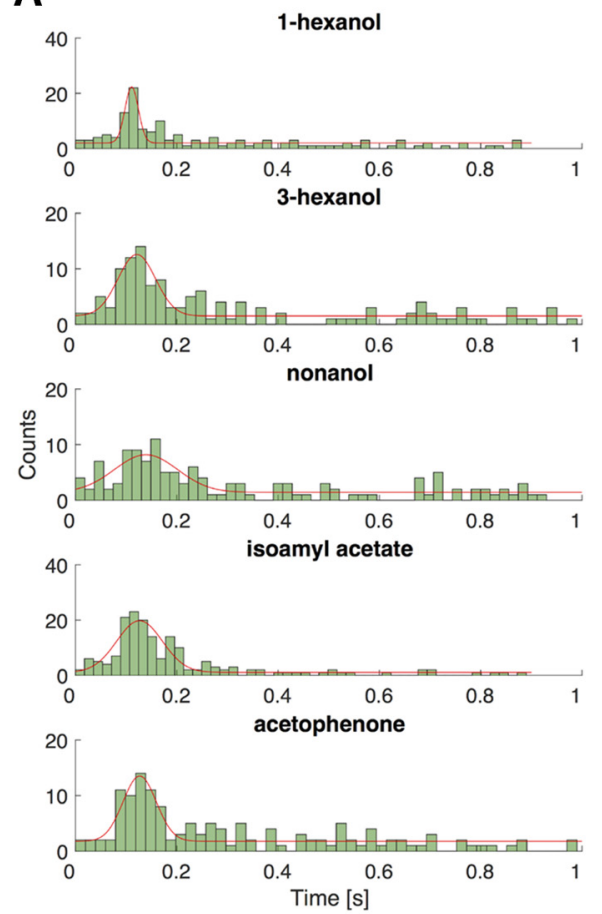

B
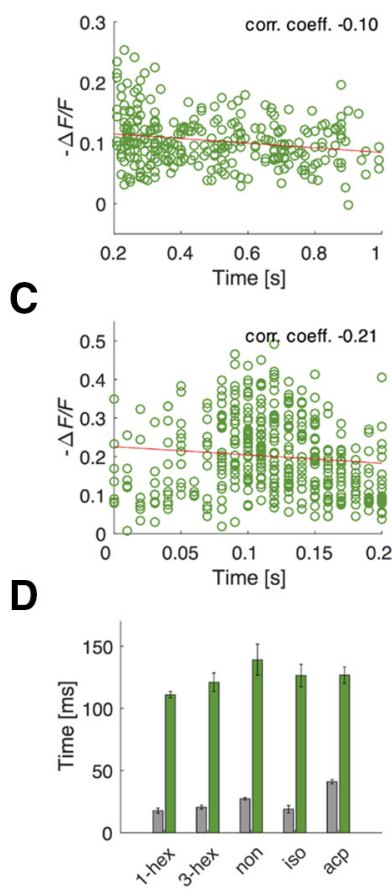

E
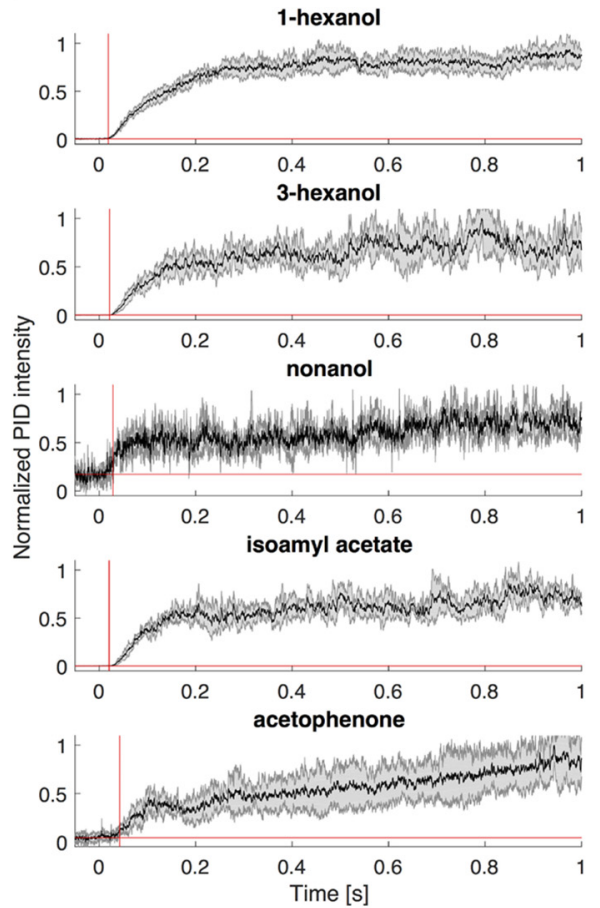

Figure 3. Quantitative analysis of response onset latency and amplitude distribution. $A$, Histograms of the response latency distribution across all active glomeruli in all tested animals for the five odorants. Gaussian fits mark the peaks of the early evoked responses. $\boldsymbol{B}$, Plot of the early $(<200 \mathrm{~ms})$ glomerular response latencies, pooled across all bees and odorants, versus the corresponding response amplitudes, measured by the relative fluorescence change (Pearson's correlation coefficient, -0.10$)$. C, Plot of the late ( $>200 \mathrm{~ms}$ ) glomerular response latencies, pooled across all bees and all tested odorants, versus the corresponding response amplitudes (Pearson's correlation coefficient, -0.21 ). $\boldsymbol{D}$, Mean latency of the evoked responses (peaks of the normal distribution in $\boldsymbol{A}$; green bars) compared with the delay of the odor arrival at the position of the bee's antennae measured by PID (gray bars). 1-hex, 1-Hexanol; 3-hex, 3-hexanol; non, nonanol; iso, isoamyl acetate; acp, acetophenone. $E$, PID response curves for the five odorants (mean \pm SEM $n=5$ ). Red lines denote threshold criteria and onset points. $-\Delta F / F$ indicates the change in fluorescence normalized to background fluorescence. corr. coeff., Correlation coefficient.

clearance of intracellular calcium limit the temporal resolution of response offset (Galizia and Kimmerle, 2004; Moreaux and Laurent, 2007; Grewe et al., 2010).

Response latencies were found to be broadly distributed (Fig. $3 A$ ). The distribution contains two components, one uniformly distributed over the whole stimulus duration and the other one normally distributed, with short latencies $\sim 125 \mathrm{~ms}$. To test whether response latencies and amplitudes provide redundant measures of the same quantity, we analyzed the amplitude-latency correlation of all responses in both distribution components. This analysis detected little or no correlation between both degrees of freedom of the response patterns, with Pearson's correlation coefficients of -0.10 and -0.21 for the early ( $<200 \mathrm{~ms}$ ) evoked responses and the late $(>200 \mathrm{~ms})$ responses, respectively (Fig. $3 B, C$ ). To further investigate how fast PNs respond to an olfactory stimulus, we measured odorant arrival times at the bee antennae (Fig. 3E) and compared them with the peaks of the evoked response distributions observed in Figure $3 A$. Notably, odor delivery kinetics was highly reproducible and odorant specific, showing onsets between $18 \pm 2 \mathrm{~ms}(1$-hexanol) and $41 \pm 2$ $\mathrm{ms}$ (acetophenone) after valve opening (Fig. $3 D$, gray bars). To estimate the absolute response latencies, the differences between odor stimulus onsets and mean neuronal activation latencies were calculated, varying between $86 \mathrm{~ms}$ for acetophenone and $112 \mathrm{~ms}$ for 1-nonanol. Therefore, first sensory information arrives, on average, after $100 \mathrm{~ms}$ from stimulus delivery.

\section{Odorant prediction from the latency rank code}

A full analysis of a potential latency code is often hindered by fluctuations caused by odorant-independent offsets (e.g., an- tennal movements, oscillatory pattern cycles, and individual network variability) and glomerulus-independent offsets (e.g., differences in volatility of the single odorants). To account for these effects, for each animal we built a vector, where glomerular responses were ranked based on their response latencies, that is the sequence with which responsive glomeruli start firing. In this way, the response of an individual animal to a certain odorant is represented by a latency rank vector, composed of the glomerular labels in the order of their response onsets (see Materials and Methods for details). The similarity between latency rank vectors was quantified by Kendall's rank correlation coefficient $\tau$ (Kendall, 1938), which provides a measure for the odorant specificity of a latency rank code across individuals. Importantly, both stimulus-evoked signals and spontaneous activity contributed to the latency rank vector for a certain odorant in a given individual. Hence, the predictability of a latency-based code is challenged by the presence of random noise.

To probe the universality of a latency rank code, we calculated the correlation between response latency rank vectors of individual "test" bees with template vectors obtained by averaging the response latencies of all remaining animals to a specific odorant. As a classifier for blind identification of the test odorant, we used the maximum rank correlation coefficient $\tau_{\max }$ between the test latency vector and the five odor template vectors. To quantify odor prediction accuracy, each bee was selected once as a test subject, providing 25 tests for each odorant. Figure $4 \mathrm{~A}$ shows a latency code prediction matrix, where the prediction probability for each template odorant is shown for every test odorant. 
A

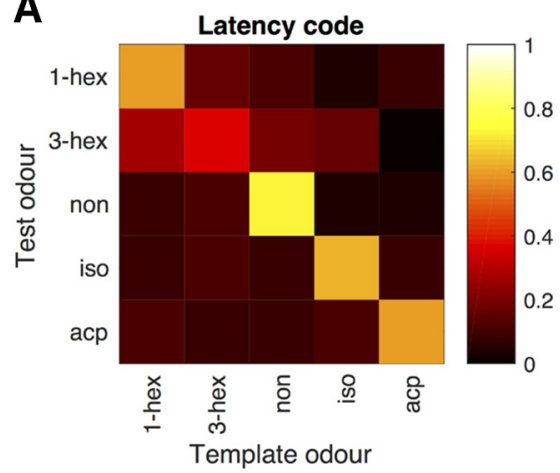

D

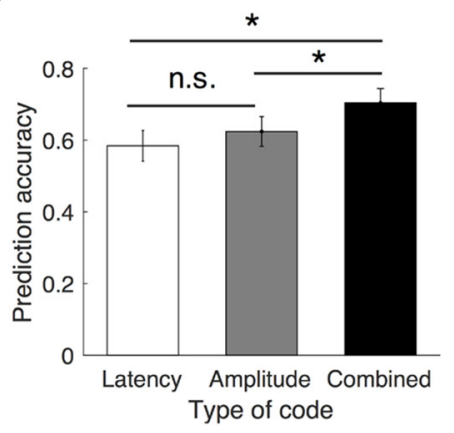

B

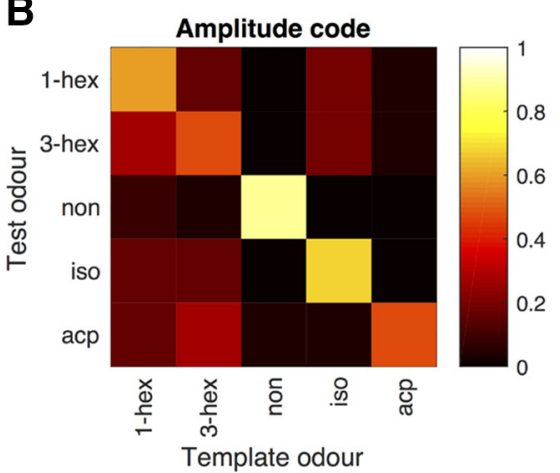

E

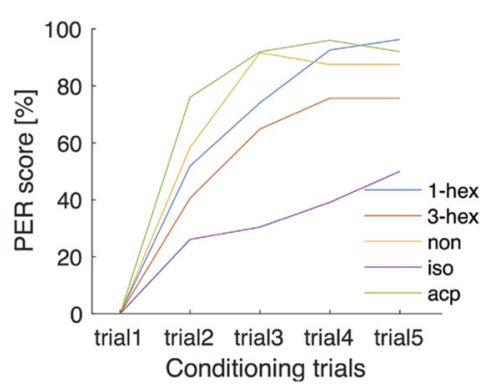

C

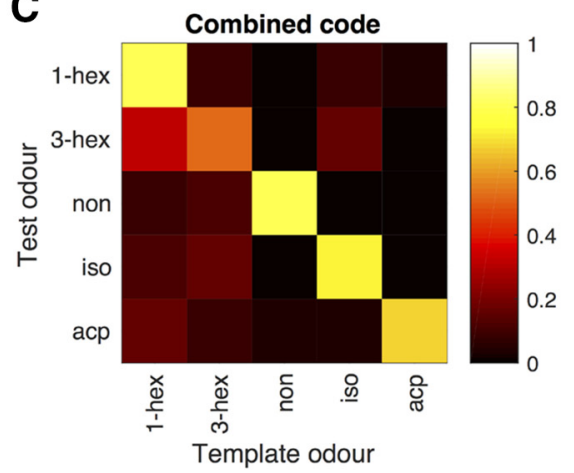

$\mathbf{F}$

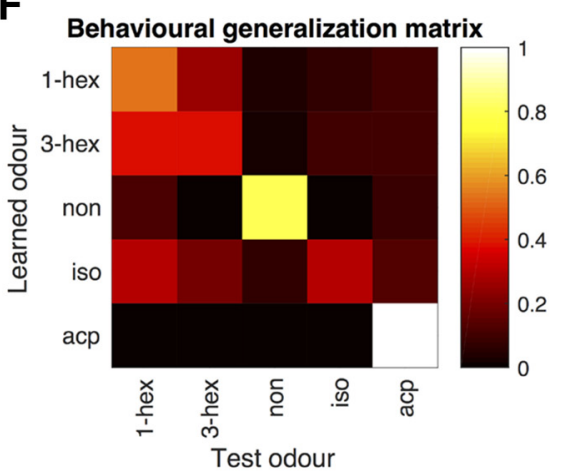

Figure 4. Odorant prediction accuracy of rank latency vectors. $\boldsymbol{A}-\boldsymbol{C}$, Generalization matrices of the three neural odor codes based on glomerular latencies $(\boldsymbol{A})$, amplitudes $(\boldsymbol{B})$, and combined codes (C). Each matrix shows the probability of a test odorant being identified as one of the five odor templates (based on $n=25$ test bees). $\boldsymbol{D}$, The combined code performs significantly better than the latency $(p=0.011)$ and amplitude $(p=0.033)$ codes. No difference was found between amplitude and latency code prediction accuracy ( $p=0.51$, all paired $t$-test, Benjamini-Hochbergcorrected; * indicates $p$ values $<0.05$ ). $\boldsymbol{E}$, Learning curves show the improvement in the PER response percentage to the CS across five conditioning trials. The number of conditioned animals for the five odorants were as follows: 1-hex, $n=27 ; 3$-hex, $n=37$; non, $n=34$; iso, $n=46$; acp, $n=25$. F, Generalization matrix obtained from the psychometric data of the PER assay. The matrix shows the proboscis extension probabilities for all combinations of conditioned/tested odors. Only animals that responded to at least one test odor were considered. For the five groups, the number of individuals was as follows: 1-hex, $n=26$; 3-hex, $n=26$; non, $n=29$; iso, $n=23$; acp, $n=25$. 1-hex: 1-Hexanol; 3-hex, 3-hexanol; non, nonanol; iso, isoamyl acetate; acp, acetophenone.

$\chi^{2}$ tests rejected the null hypothesis that predictions were random for all test odors except for 3-hexanol [1-hexanol: $\chi^{2}(4)=$ 25.2, $p=0.0002$; 3-hexanol: $\chi^{2}(4)=6.8, p=0.7342$; 1-nonanol: $\chi^{2}(4)=38.2, p=0$; isoamyl acetate: $\chi^{2}(4)=31.6, p=0$; acetophenone: $\chi^{2}(4)=31.8, p=0$ ]. In four of five odorants, the correct test/template combination was strongly dominant, showing a prediction accuracy between 60 and $70 \%$, which is significantly above the $20 \%$ chance level. This suggests that the latency rank code provided sufficient information for odorant recognition. A lower percentage of correct predictions was observed for 3-hexanol, a position isomer of 1-hexanol with very high structural similarity. In fact, 3-hexanol was correctly recognized in $36 \%$ of the cases, whereas it was confused with 1-hexanol in $28 \%$ of the cases, accounting for the majority of all prediction errors.

\section{Comparison with amplitude code and combined code}

Next, we assessed the accuracy of the intersubject odor prediction with respect to the commonly used glomerular response amplitude code. From the imaging data, we extracted the amplitude rank code represented by the ordered response amplitudes of all activated glomeruli in every measurement. As for the latency code, we studied odorant specificity and universality of the amplitude code based on Kendall's rank correlation coefficient. The predictability matrix of the amplitude rank code (Fig. $4 B$ ) shows a highly similar pattern to the latency rank code, with a clear domination of the correct predictions. The correct identification probabilities are slightly higher than those of the latency code, except for acetophenone, which is correctly recognized only in $48 \%$ of the cases (vs $60 \%$ of the latency rank code). The correct identification of 3-hexanol was increased to $48 \%$, still with the highest generalization probability of $28 \%$ with 1 -hexanol.

To test how redundant the information provided by the two different features was, latency- and amplitude-based codes were combined into a single matrix. The resulting measure showed improved predictions rates compared with the two separate codes (Fig. 4C). Although acetophenone was correctly predicted in $68 \%$ of the cases, the similarity between 3 -hexanol and 1-hexanol still challenged the predictive power of the code. In fact, whereas 3 -hexanol is correctly matched in $52 \%$ of the cases, the same odorant is confused with 1-hexanol in $32 \%$ of the cases.

Finally, we quantified the overall performance of the two codes by calculating an average prediction accuracy across odorants (58 $\pm 5,62 \pm 5$, and $70 \pm 4 \%$ for latency, amplitude, and combined code, respectively; Fig. 4D). A statistical analysis showed that the provided information is not significantly different between the two single-feature codes $\left(t_{(124)}=-0.663, p=\right.$ $0.51)$. Instead, the combined code provides significantly more odor-related information compared with the latency code $\left(t_{(124)}=-2.60, p=0.011\right)$ or the amplitude code $\left(t_{(124)}=-2.17\right.$, $p=0.033$; all results are corrected for a false discovery rate via the Benjamini-Hochberg method; Fig. 4D).

\section{Comparison of neural codes and behavioral odor generalization}

To test to what degree the neural codes match the behavioral output, the predictability matrices based on latency, amplitude, and combined codes were compared with a behavioral assay for learned odorant recognition. All used odorants were successfully 

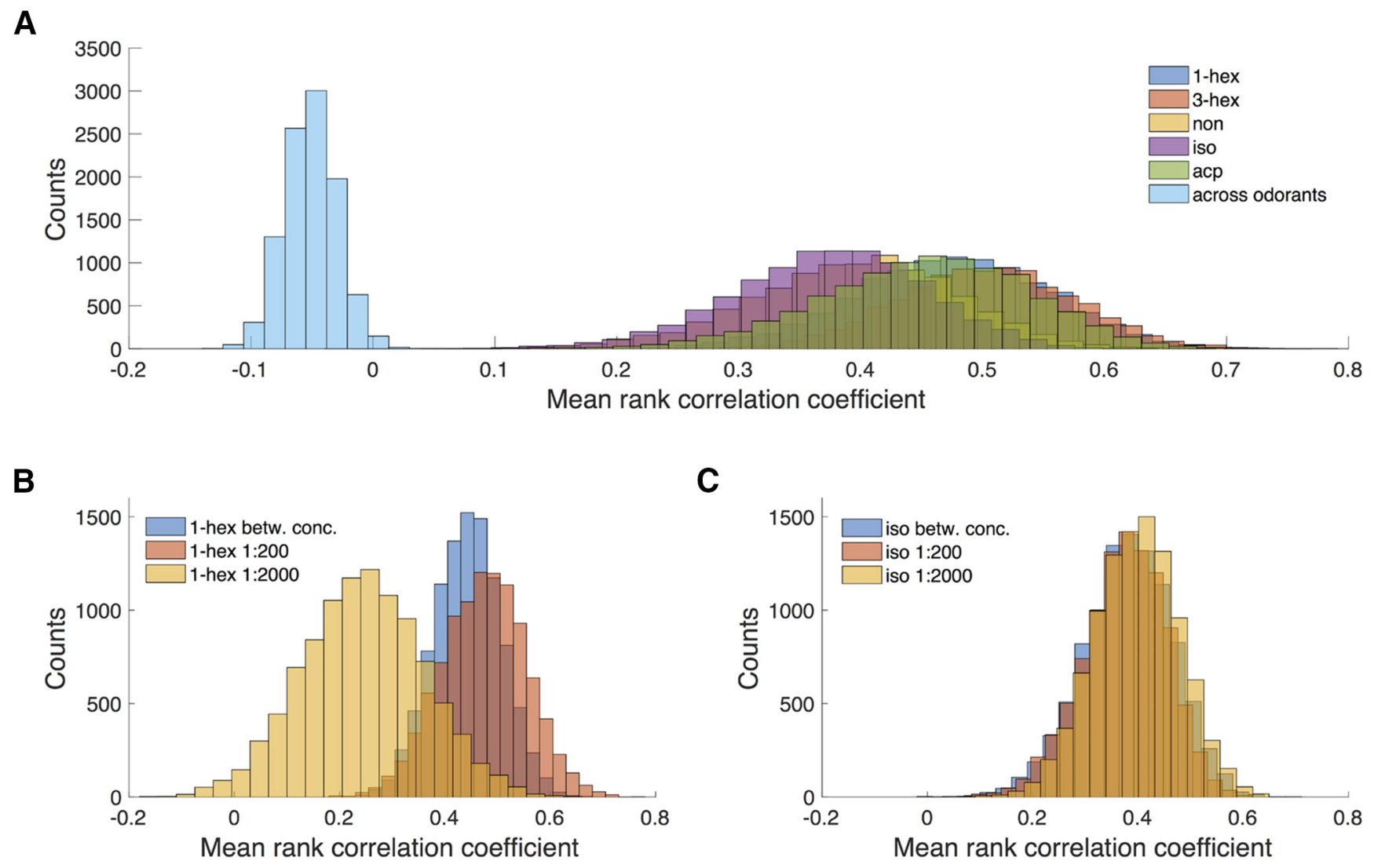

Figure 5. Bootstrap analysis with replacements on the mean rank correlation coefficient. $\boldsymbol{A}$, Bootstrap distribution of the mean correlation coefficients between responses to the same stimulus and between responses to different stimuli, showing the complete absence of correlations across different odorants. 1-hex: 1-Hexanol; 3-hex, 3-hexanol; non, nonanol; iso, isoamyl acetate; acp, acetophenone. $\boldsymbol{B}, \boldsymbol{C}$, Bootstrap distribution of the mean rank correlation coefficients between responses to low concentration, between responses to high concentration, and across the different concentrations. 1-Hexanol (1-hex, $\boldsymbol{B}$ ) and isoamyl acetate (iso, $\boldsymbol{C}$ ) were used as odorants.

learned by the bees, and for all but isoamyl acetate, a PER score of $>75 \%$ was reached at the fifth conditioning trial. Associative conditioning with isoamyl acetate was less successful, providing a final score of $52 \%$ (Fig. $4 E$ ). After conditioning, bees were tested for generalization effect by sequentially probing each animal with all five odorants (including the learned one) in the absence of a sugar reward. A positive PER response would show the recognition of the learned odorant or, if the test odorant was a novel one, a generalization effect. In the following sections, we name the resulting response probabilities as the behavioral generalization matrix, although effects likewise may be caused by the inability to discriminate between learned and tested odorants. The behavioral generalization matrix (Fig. $4 F$, off-diagonal data) shows the PER probability in the presence of odorants different from the one learned during training, normalized by the responses to all test odorants. The highest response probability was always observed after presentation of the conditioned stimulus, whereas a lower degree of responsiveness was shown when bees were presented with novel odorants. However, an identical PER score for both isomers suggests that bees either generalize between the two odorants or failed to discriminate them. Instead, bees trained on isoamyl acetate showed a generally higher degree of generalization that seemed independent of the odorants' chemical similarity. Multiple $t$ tests confirmed the preference for the trained odorant with respect to all other odorants for bees conditioned to 1-hexanol, acetophenone, and 1-nonanol [12 odor pair combinations, maximum $p=1.0 \times 10^{-7}$ for (1-hex/3-hex), Bonferroni corrected]. The response frequencies to 1 -hexanol and 3-hexanol were not significantly different when animals were conditioned with 3-hexanol. Similarly, response frequencies to 1-hexanol and isoamyl acetate did not differ for bees conditioned with isoamyl acetate.

To quantify the odor generalization predicted by the neuronal codes and the behavioral output, we compared the matrix elements of the three different codes (Fig. $4 A-C$ ) with the PER assaybased generalization matrix (Fig. $4 F$ ) with a $\chi^{2}$ goodness-of-fit test. The results show a high agreement of neural code and behavioral data: $\chi^{2}(18)=3.13$ and $p=3.8 \times 10^{-5}$ for latency coding, $\chi^{2}(18)=1.73$ and $p=3.5 \times 10^{-7}$ for amplitude coding, and $\chi^{2}(18)=1.61$ and $p=1.9 \times 10^{-7}$ for the combined code.

\section{Concentration independence of the latency rank code}

Finally, we investigated whether the latency rank vector analysis could also discriminate between different odorant concentrations. Thus, we compared the response with two odorants, 1-hexanol and isoamyl acetate, at different dilutions: a lower one, $1: 2000$, and a higher one, 1:200, as used in the previous experiments. We calculated the mean rank correlation coefficients across subjects (Eq. 6) and looked at their distribution via a bootstrap analysis. When performing this analysis on the five odorants at the same concentration, the correlations within single odor groups were comparably high, whereas no correlation was found when comparing responses with different odorants (Fig. $5 A$ ). In particular, the highest mean latency rank correlation across different odorants was smaller than 0.02 , whereas the lowest correlation within an odor group was higher than 0.08 . This 
shows that random correlations (e.g., attributable to spontaneous activity in single bees) are unlikely to significantly influence the stimulus-induced correlations.

We then studied mean latency ranks across stimulus concentrations. The mean rank correlation coefficients computed for 1-hexanol at low concentration (average, 0.25 ) were strongly reduced compared with the high concentration-induced ones (average, 0.47), indicating a weak correlation at a low odorant concentration. In addition, the correlation between responses to low- and high-concentration odorants (average, 0.44) was found to be higher than the correlation of neural responses to the lowconcentration stimulus (Fig. 5B). For isoamyl acetate, the mean rank correlation coefficient distribution was not significantly lower for the low concentration (average, 0.40) with respect to the high concentration (average, 0.37 ). However, the correlation between low- and high-concentration responses was found to be equally high (mean, 0.38 ; Fig. $5 C$ ).

\section{Discussion}

The question of how odor information is encoded within the insect antennal lobe, or the vertebrate olfactory bulb, has been widely investigated. A major finding has been the observation that odorants are represented in the glomerular space by glomerular response maps based on the mean response amplitudes of individual glomeruli. This code was found to be highly conserved across animals (Galizia et al., 1999b). Furthermore, it was shown that temporal features of the glomerular responses are also carrying information on the odor stimulus identity as well as its intensity (Stopfer et al., 2003).

Here, we investigate a coding parameter that may provide the first information on an olfactory stimulus, the glomerular response latencies. Our aim is to clarify the following fundamental open questions about a potential latency code: 1) Are stimulusspecific latency patterns conserved across individuals? 2) Can the latency code compete with the static amplitude code in odor discrimination power? 3) Do glomerular latencies and amplitudes carry complementary information? 4) Is the latency rank code capable of distinguishing between different odorant concentrations? 5) Can the latency code predict behavioral odor discrimination performance?

Notably, the fundamental electrophysiological studies that provided first insights into the temporal dimension of odor coding focused on oscillatory features over an extended stimulus duration. Their analysis excluded the fast dynamics by restricting time resolution to $50 \mathrm{~ms}$ (Laurent and Davidowitz, 1994; Wehr and Laurent, 1996; Stopfer et al., 2003; Mazor and Laurent, 2005).

Here, we adopted a complementary approach based on a fast calcium imaging analysis, which allows detecting both evoked and spontaneous activities with high temporal resolution (Moreaux and Laurent, 2007; Grewe et al., 2010). Indeed, a small subset of the detected responses is likely attributable to spontaneous firing (Galán et al., 2006; Grewe et al., 2010). However, we did not remove these events from the analysis because spontaneous activities can be distinguished from stimulus-evoked activities only in terms of duration, not by onset timing. Notably, higher-order brain centers that might read out the latency code will also face the same problem (Jortner et al., 2007). Thus, we decided to analyze the latency code in the presence of this background activity, to test its robustness under naturally noisy conditions.

A limitation of calcium imaging analysis is that detectable intracellular calcium increase displays a delay of a few millisec- onds with respect to changes in voltage potential (Homma et al., 2009; Junek et al., 2010). Nonetheless, this aspect would influence an analysis of absolute latency values but not the response latency ranks.

This study extends an analysis of response latencies across subjects. Although the labeling method is susceptible to acrosssubject variability, e.g., the number of labeled PNs or the amount of incorporated dye, this did not significantly alter kinematics nor amplitude of measured response curves (see Fig. 2-1, available at https://doi.org/10.1523/JNEUROSCI.0453-18.2018.f2-1; and for a corresponding 4D data array, see Fig. 2-2, available at https://doi.org/10.1523/JNEUROSCI.0453-18.2018.f2-2). Also, previous calcium imaging studies on $\mathrm{PN}$ activity revealed a high level of synchronization within each glomerulus (Franke, 2009), suggesting that a variable number of labeled PNs within a given glomerulus is not influential on the measurement of the relative response onset.

To test the predictive power of the latency code, we selected five odorants with very different significance for honeybees: floral compounds like alcohols (1-hexanol, 3-hexanol, and 1-nonanol); aromatic ketones (acetophenone); and a component of the sting alarm pheromone, like the ester isoamyl acetate (Boch et al., 1962). To further challenge the code's predictive power, some components were chosen to be structurally similar, like 1-hexanol and 1-nonanol, or almost identical, such as 1-hexanol and 3-hexanol. The analysis was conducted on a population of 25 honeybees and showed that the response latency rank patterns allowed distinguishing all odorants with statistical significance, except for the position isomers (i.e., 1-hexanol and 3-hexanol). In all significant cases, the prediction accuracy was higher or equal to $60 \%$ (vs the chance level of $20 \%$ ), which is surprising given that tested individuals were not preselected based on physiological parameters such as age or foraging experience. In the only nonsignificant case of 3-hexanol, the prediction accuracy was $36 \%$, with the majority of the wrong classifications attributable to a mismatch with the 1-hexanol template. This does not necessarily imply that a high structural similarity constitutes a limit for the latency code. Most likely, this limitation derives from the small subset of active glomeruli comparable across all animals. In fact, we expect that increasing the number of involved glomeruli would also increase the code's predictive power. Junek et al. (2010) performed a similar analysis, although limited to single subjects, where they monitored the neural responses of the olfactory bulb in individual Xenopus tadpoles. They reported that the prediction accuracy for a set of three odorants increased together with the number of activated neurons, from $70 \%$ accuracy for three responding cells to $100 \%$ accuracy when 10 neurons or more were activated. Although the present study adds additional complexity by considering across-subject variations, the numbers provided by Junek et al. (2010) are in agreement with our vector correlation analysis, which was usually limited to an average of five to six commonly active glomeruli per comparison. The present findings also indicate that the temporal features are conserved and allow odor prediction across individuals.

Next, we compared the latency rank code with the amplitude rank code, detecting no significant difference between the average predictive power of the two separate codes. To quantify the redundancy between codes, we performed a correlation analysis between response latencies and amplitudes across all measured responses. The low correlation coefficients (Fig. $3 B, C$ ) indicate that the two parameters are not correlated, suggesting that they may provide different and complementary information. Indeed, a combined code based on both features provided a significant 
increase in predictive power with respect to both the latency and the amplitude-based codes. This suggests that the temporal and spatial features of the odor response patterns carry complementary information. These findings are in agreement with previously reported measurements of the response latencies and amplitudes of mice ORNs (Spors et al., 2006), where no correlation was found.

How stimulus properties are actually encoded within the spatiotemporal response patterns is still debated. Carcaud et al. (2015) observed that the pheromonal compound of honeybee queens, drones, or workers were processed differently by the projection neurons of m-ACTs and l-ACTs. More recently, the same authors suggested that parallel processing could also affect common odorant processing, with the two projection neuron tracts delivering different and complementary information on the nature of the received odorants (Carcaud et al., 2015, 2018). Brill et al. (2013) simultaneously performed electrophysiological recordings on the m-ACTs and l-ACTs of the honeybee AL output neurons and observed that the more broadly tuned l-ACT delivers fast general information on odor timing, whereas the medial tract relays on more specific information of odor identity, although with a temporal offset. In our imaging experiments, we recorded exclusively from l-ACT PNs and were able to confirm the fast and heterogeneous response time distribution reported previously. In addition, we could show that the fast responses detected in the 1-ACT PNs do not simply depend on the stimulus dynamics but are both glomerulus and stimulus specific and encode odor identity information.

Can the response latency rank code provide information also on the odorant's concentration? A direct comparison of the correlations between responses obtained after stimulation with high and low odor concentrations advocates for a concentration independence of the latency rank code. A previous study suggested that glomerular response latencies might provide a concentration-invariant code for prompt odor identification (Krofczik et al., 2008), a code later complemented by the average firing rate (i.e., the amplitude code) conveying concentrationrelated information. This view is further supported by a study in Drosophila, where a complete concentration invariance of the ORNs dynamics was observed (Martelli et al., 2013). Instead, a weak concentration dependency of response latencies has previously been shown in the mitral/tufted cells in the Xenopus olfactory bulb (Junek et al., 2010) and in the initial response dynamics of the rat olfactory sensory neurons (Carey et al., 2011). Importantly, when analyzing concentration dependence, possible differences between the information content of absolute response latencies and relative latency ranks need to be considered. With increasing stimulus concentration, a decrease in absolute response latency can be expected because of an increase in odorantreceptor interaction probability (Egea-Weiss et al., 2018). Indeed, a neuronal network model of pheromone coding in moths proposed that differences in relative latency encode concentration-invariant information on stimulus identity, whereas absolute latencies reflect stimulus intensity (Belmabrouk et al., 2011). Similarly, an imaging study in the rat and mouse olfactory bulb also showed that increasing odor concentration resulted in reduced absolute response latencies, whereas the sequence of glomerular activation was unaffected (Spors and Grinvald, 2002).

Finally, we compared the classification performance of the latency rank code with the honeybees' behavioral generalization between a learned odorant and a set of different test compounds. All tests showed a generally high differentiation efficiency and a low level of generalization for chemically diverse odorants. In- stead, a strong generalization was observed for 1-hexanol and 3-hexanol, as predicted by the latency rank code. Contrary to the predictability codes, the behavioral response of isoamyl acetatetrained animals turned out to be more complex. As reported previously, isoamyl acetate appeared to be more difficult to learn compared with other odorants (Wang et al., 2016). This odorant is a component of the bee alarm pheromone, which may modulate honeybee neurophysiology and explain the reported results (Urlacher et al., 2010; Baracchi et al., 2017; Nouvian et al., 2018). Overall, behavioral tests were coherent with an odorant classification model based on the glomerular response latency rank code. Although strong differences in the latency rank code correctly predict a clear distinction between test odorants, the higher similarity between latency rank vectors of similar odors is confirmed by a high degree of generalization in the behavioral test. The quantitative analysis of the correct prediction of behavioral responses by the glomerular code was performed via a goodnessof-fit test between the neural code-based predictions and the behavioral generalization matrix elements. The results reflect the high agreement between all codes and the behavior, where the highest significance level is reached by the combined code, suggesting that odor information stored in the latency ranks might indeed contribute to the control of motor output.

In conclusion, this study provides the first experimental evidence that glomerular response latencies are conserved across individuals. Consequently, a code based on the glomerular response latency rank allows odorant identification across subjects, with similar prediction accuracy as the glomerular response amplitude code. Furthermore, the latency rank code appears to be concentration invariant, thus supporting the idea that it provides a fast mechanism to encode odorant identity, which is then complemented by slower features providing odor intensity information. Indeed, a comparison of the predictive power of a code based on amplitude, latency, or both features showed that a combined code has a significantly greater prediction accuracy, supporting the hypothesis that the odor perception may rely on both a fast/temporal and a slow/spatial component.

\section{References}

Abraham NM, Spors H, Carleton A, Margrie TW, Kuner T, Schaefer AT (2004) Maintaining accuracy at the expense of speed: stimulus similarity defines odor discrimination time in mice. Neuron 44:865-876. CrossRef Medline

Baracchi D, Devaud JM, d'Ettorre P, Giurfa M (2017) Pheromones modulate reward responsiveness and non-associative learning in honey bees. Sci Rep 7:9875. CrossRef Medline

Bathellier B, Buhl DL, Accolla R, Carleton A (2008) Dynamic ensemble odor coding in the mammalian olfactory bulb: sensory information at different timescales. Neuron 57:586-598. CrossRef Medline

Belmabrouk H, Nowotny T, Rospars JP, Martinez D (2011) Interaction of cellular and network mechanisms for efficient pheromone coding in moths. Proc Natl Acad Sci U S A 108:19790-19795. CrossRef Medline

Bitterman ME, Menzel R, Fietz A, Schäfer S (1983) Classical conditioning of proboscis extension in honeybees (Apis mellifera). J Comp Psychol 97: 107-119. CrossRef Medline

Boch R, Shearer DA, Stone BC (1962) Identification of iso-amyl acetate as an active component in the sting pheromone of the honey bee. Nature 195:1018-1020. CrossRef

Brill MF, Rosenbaum T, Reus I, Kleineidam CJ, Nawrot MP, Rössler W (2013) Parallel processing via a dual olfactory pathway in the honeybee. J Neurosci 33:2443-2456. CrossRef Medline

Carcaud J, Giurfa M, Sandoz J-C (2015) Differential combinatorial coding of pheromones in two olfactory subsystems of the honey bee brain. J Neurosci 35:4157-4167. CrossRef Medline

Carcaud J, Giurfa M, Sandoz J-C (2018) Differential processing by two olfactory subsystems in the honeybee brain. Neuroscience 374:33-48. CrossRef Medline 
Carey RM, Verhagen JV, Wesson DW, Pírez N, Wachowiak M (2011) Temporal structure of receptor neuron input to the olfactory bulb imaged in behaving rats. Stimulus 101:1073-1088. CrossRef Medline

Chase SM, Young ED (2007) First-spike latency information in single neurons increases when referenced to population onset. Proc Natl Acad Sci U S A 104:5175-5180. Medline

Egea-Weiss A, Renner A, Kleineidam CJ, Szyszka P (2018) High precision of spike timing across olfactory receptor neurons allows rapid odor coding in Drosophila. iScience 4:P76-P83. CrossRef

Franke T (2009) In vivo 2-photon calcium imaging of olfactory interneurons in the honeybee antennal lobe. PhD thesis, FU Berlin.

Furukawa S, Middlebrooks JC (2002) Cortical representation of auditory space: information-bearing features of spike patterns. J Neurophysiol 87: 1749-1762. CrossRef

Galán RF, Weidert M, Menzel R, Herz AV, Galizia CG (2006) Sensory memory for odors is encoded in spontaneous correlated activity between olfactory glomeruli. Neural Comput 18:10-25. CrossRef Medline

Galizia CG (2014) Olfactory coding in the insect brain: data and conjectures. Eur J Neurosci 39:1784-1795.

Galizia CG, Kimmerle B (2004) Physiological and morphological characterization of honeybee olfactory neurons combining electrophysiology, calcium imaging and confocal microscopy. J Comp Physiol A Neuroethol Sens Neural Behav Physiol 190:21-38. Medline

Galizia CG, McIlwrath SL, Menzel R (1999a) A digital 3-dimensional atlas of the honeybee antennal lobe based on optical sections acquired using confocal microscopy. Cell Tissue Res 295:383-394. CrossRef

Galizia CG, Sachse S, Rappert A, Menzel R (1999b) The glomerular code for odor representation is species specific in the honeybee Apis mellifera. Nat Neurosci 2:473-478. CrossRef Medline

Gawne TJ, Kjaer TW, Richmond BJ (1996) Latency: another potential code for feature binding in striate cortex. J Neurophysiol 76:1356-1360. CrossRef Medline

Giurfa M, Sandoz J-C (2012) Invertebrate learning and memory: fifty years of olfactory conditioning of the proboscis extension response in honeybees. Learn Mem 19:54-66. CrossRef Medline

Grewe BF, Langer D, Kasper H, Kampa BM, Helmchen F (2010) High-speed in vivo calcium imaging reveals neuronal network activity with nearmillisecond precision. Nat Methods 7:399-405. CrossRef Medline

Grillet M, Campagner D, Petersen R, McCrohan C, Cobb M (2016) The peripheral olfactory code in Drosophila larvae contains temporal information and is robust over multiple timescales. Proc R Soc Lond B Biol Sci 283:20160665. CrossRef Medline

Guerrieri F, Schubert M, Sandoz JC, Giurfa M (2005) Perceptual and neural olfactory similarity in honeybees. PLoS Biol 3:e60. CrossRef Medline

Haase A (2011) Simultaneous morphological and functional imaging of the honeybee's brain by two-photon microscopy. Nuovo Cimento della Societa Italiana di Fisica C 34:165-174. CrossRef

Haase A, Rigosi E, Frasnelli E, Trona F, Tessarolo F, Vinegoni C, Anfora G, Vallortigara G, Antolini R (2011a) A multimodal approach for tracing lateralisation along the olfactory pathway in the honeybee through electrophysiological recordings, morpho-functional imaging, and behavioural studies. Eur Biophys J 40:1247-1258. CrossRef Medline

Haase A, Rigosi E, Trona F, Anfora G, Vallortigara G, Antolini R, Vinegoni C (2011b) In-vivo two-photon imaging of the honey bee antennal lobe. Biomed Opt Express 2:131. CrossRef Medline

Homma R, Baker BJ, Jin L, Garaschuk O, Konnerth A, Cohen LB, Bleau CX, Canepari M, Djurisic M, Zecevic D (2009) Wide-field and two-photon imaging of brain activity with voltage- and calcium-sensitive dyes. Methods Mol Biol 489:43-79. CrossRef Medline

Hopfield JJ (1995) Pattern recognition computation using action potential timing for stimulus representation. Nature 376:33-36. CrossRef

Jortner RA, Farivar SS, Laurent G (2007) A simple connectivity scheme for sparse coding in an olfactory system. J Neurosci 27:1659-1669. CrossRef

Junek S, Kludt E, Wolf F, Schild D (2010) Olfactory coding with patterns of response latencies. Neuron 67:872-884. CrossRef Medline

Kendall MG (1938) A new measure of rank correlation. Biometrika 30:8193. CrossRef

Krofczik S, Menzel R, Nawrot MP (2008) Rapid odor processing in the honeybee antennal lobe network. Front Comput Neurosci 2:9. CrossRef Medline

Laurent G, Davidowitz H (1994) Encoding of olfactory information with oscillating neural assemblies. Science 265:1872-1875. CrossRef
Laurent G, Wehr M, Davidowitz H (1996) Temporal representations of odors in an olfactory network. J Neurosci 16:3837-3847. CrossRef Medline

Margrie TW, Schaefer AT (2003) Theta oscillation coupled spike latencies yield computational vigour in a mammalian sensory system. J Physiol 546:363-374. CrossRef

Martelli C, Carlson JR, Emonet T (2013) Intensity invariant dynamics and odor-specific latencies in olfactory receptor neuron response. J Neurosci 33:6285-6297. CrossRef

Masquelier T (2012) Relative spike time coding and STDP-based orientation selectivity in the early visual system in natural continuous and saccadic vision: a computational model. J Comput Neurosci 32:425-441. CrossRef

Mazor O, Laurent G (2005) Transient dynamics versus fixed points in odor representations by locust antennal lobe projection neurons. Neuron 48: 661-673. CrossRef

Mombaerts P, Wang F, Dulac C, Chao SK, Nemes A, Mendelsohn M, Edmondson J, Axel R (1996) Visualizing an olfactory sensory map. Cell 87:675-686. CrossRef Medline

Moreaux L, Laurent G (2007) Estimating firing rates from calcium signals in locust projection neurons in vivo. Front Neural Circuits 1:2. CrossRef Medline

Müller D, Abel R, Brandt R, Zöckler M, Menzel R (2002) Differential parallel processing of olfactory information in the honeybee, Apis mellifera $\mathrm{L}$. J Comp Physiol A Neuroethol Sens Neural Behav Physiol 188:359-370. Medline

Münch D, Galizia CG (2016) DoOR 2.0-comprehensive mapping of Drosophila melanogaster odorant responses. Sci Rep 6:21841. CrossRef

Nelken I, Chechik G, Mrsic-Flogel TD, King AJ, Schnupp JW (2005) Encoding stimulus information by spike numbers and mean response time in primary auditory cortex. J Comput Neurosci 19:199-221. CrossRef Medline

Nouvian M, Mandal S, Jamme C, Claudianos C, d'Ettorre P, Reinhard J, Barron AB, Giurfa M (2018) Cooperative defense operates by social modulation of biogenic amine levels in the honeybee brain. Proc R Soc Lond B Biol Sci 285:20172653. CrossRef Medline

Panzeri S, Petersen RS, Schultz SR, Lebedev M, Diamond ME (2001) The role of spike timing in the coding of stimulus location in rat somatosensory cortex. Neuron 29:769-777. CrossRef Medline

Paoli M, Anesi A, Antolini R, Guella G, Vallortigara G, Haase A (2016a) Differential odour coding of isotopomers in the honeybee brain. Sci Rep 6:21893. CrossRef

Paoli M, Weisz N, Antolini R, Haase A (2016b) Spatially resolved timefrequency analysis of odour coding in the insect antennal lobe. Eur J Neurosci 44:2387-2395. CrossRef Medline

Paoli M, Andrione M, Haase A (2017) Imaging techniques in insects. In: Neuromethods (Rogers LJ, Vallortigara G, eds), pp 471-519. New York, NY: Springer.

Petersen RS, Panzeri S, Diamond ME (2001) Population coding of stimulus location in rat somatosensory cortex. Neuron 32:503-514. CrossRef

Petersen RS, Panzeri, Diamond ME (2002) The role of individual spikes and spike patterns in population coding of stimulus location in rat somatosensory cortex. Biosystems 67:187-193. CrossRef

Quian Quiroga R, Panzeri S (2009) Extracting information from neuronal populations: information theory and decoding approaches. Nat Rev Neurosci 10:173-185. CrossRef

Reich DS Mechler F, Victor JD (2014) Temporal coding of contrast in primary visual cortex: when, what, and why. J Neurophysiol 85:1039-1050. CrossRef Medline

Ressler KJ, Sullivan SL, Buck LB (1994) Information coding in the olfactory system: evidence for a stereotyped and highly organized epitopic map in the olfactory bulb. Cell 79:1245-1255. CrossRef

Rigosi E, Frasnelli E, Vinegoni C, Antolini R, Anfora G, Vallortigara G, Haase A (2011) Searching for anatomical correlates of olfactory lateralization in the honeybee antennal lobes: a morphological and behavioural study. Behav Brain Res 221:290-294. CrossRef Medline

Rigosi E, Haase A, Rath L, Anfora G, Vallortigara G, Szyszka P (2015) Asymmetric neural coding revealed by in vivo calcium imaging in the honey bee brain. Proc R Soc Lond B Biol Sci 282:20142571. CrossRef Medline

Sachse S, Galizia CG (2002) Role of inhibition for temporal and spatial odor representation in olfactory output neurons: a calcium imaging study. J Neurophysiol 87:1106-1117. CrossRef

Sachse S, Galizia CG (2003) The coding of odour-intensity in the honeybee 
antennal lobe: local computation optimizes odour representation. Eur J Neurosci 18:2119-2132. CrossRef

Schaefer AT, Margrie TW (2007) Spatiotemporal representations in the olfactory system. Trends Neurosci 30:92-100. CrossRef Medline

Spors H, Grinvald A (2002) Spatio-temporal dynamics of odor representations in the mammalian olfactory bulb. Neuron 34:301-315. CrossRef

Spors H, Wachowiak M, Cohen LB, Friedrich RW (2006) Temporal dynamics and latency patterns of receptor neuron input to the olfactory bulb. J Neurosci 26:1247-1259. CrossRef Medline

Stopfer M, Jayaraman V, Laurent G (2003) Intensity versus identity coding in an olfactory system. Neuron 39:991-1004. CrossRef

Szyszka P, Demmler C, Oemisch M, Sommer L, Biergans S, Birnbach B, Silbering AF, Galizia CG (2011) Mind the gap: olfactory trace conditioning in honeybees. J Neurosci 31:7229-7239. CrossRef Medline
Takeda K (1961) Classical conditioned response in the honey bee. J Insect Physiol 6:168-179. CrossRef

Thorpe S, Delorme A, Van Rullen R (2001) Spike-based strategies for rapid processing. Neural Netw 14:715-725.

Urlacher E, Francés B, Giurfa M, Devaud JM (2010) An alarm pheromone modulates appetitive olfactory learning in the honeybee (Apis mellifera). Front Behav Neurosci 4:157. CrossRef Medline

Wang Z, Qu Y, Dong S, Wen P, Li J, Tan K, Menzel R (2016) Honey bees modulate their olfactory learning in the presence of hornet predators and alarm component. PLoS One 11:e0150399. CrossRef Medline

Wehr M, Laurent G (1996) Odour encoding by temporal sequences of firing in oscillating neural assemblies. Nature 384:162-166. CrossRef Medline

Wilson CD, Serrano GO, Koulakov AA, Rinberg D (2017) A primacy code for odor identity. Nat Commun 8:1477. CrossRef Medline 